\title{
Turnip mosaic virus (TuMV) Is Able to Use Alleles of Both elF4E and elF(iso)4E from Multiple Loci of the Diploid Brassica rapa
}

\author{
Carol E. Jenner, Charlotte F. Nellist, Guy C. Barker, and John A. Walsh \\ Warwick HRI, University of Warwick, Warwick, CV35 9EF, U.K. \\ Submitted 6 May 2010. Accepted 8 July 2010.
}

\begin{abstract}
Three copies of $e I F 4 E$ and three copies of $e I F($ iso $) 4 E$ have been identified and sequenced from a Turnip mosaic virus (TuMV)-susceptible, inbred, diploid Brassica rapa line, $\mathbf{R}$ o-18. One of the copies of $e I F 4 E$ lacked exons 2 and 3 and appeared to be a pseudogene. The two other copies of $e I F 4 E$ and two of the three copies of $e I F(i s o) 4 E$ were isolated from a bacterial artificial chromosome library of Ro-18. Using an Arabidopsis line (Col-0::dSpm) with a transposon knock-out of the $e I F$ (iso) $4 E$ gene which resulted in a change from complete susceptibility to complete resistance to TuMV, complementation experiments were carried out with the two versions of $e I F 4 E$ and the two versions of $e I F($ iso $) 4 E$. When transformed into Col-0::dSpm, all four Brassica transgenes complemented the Arabidopsis eIF(iso) $4 E$ knock-out, conferring susceptibility to both mechanical and aphid challenge with TuMV. One of the copies of $e I F 4 E$ did not appear to support viral replication as successfully as the other copy of $e I F 4 E$ or the two copies of eIF(iso)4E. The results show that TuMV can use both $e I F 4 E$ and $e I F$ (iso) $4 E$ from $B$. rapa for replication and, for the first time, that a virus can use $e I F 4 E$ and $e I F($ iso $) 4 E$ from multiple loci of a single host plant.
\end{abstract}

A key step in plant mRNA translation involves the binding of the $5^{\prime}$ cap to the $40 \mathrm{~S}$ ribosomal subunit. This is achieved partly through the interaction of the cap with the eukaryotic initiation complex eIF4F, itself a combination of the cap-binding subunit eIF4E and the larger subunit eIF4G (Browning 1996). Isoforms of each component, eIF(iso)4E and eIF(iso)4G, also exist in plants (Allen et al. 1992). It is becoming clear that most eukaryotic organisms encode and express multiple eIF4E family members, some for general translation and others for specific functions, including control of translation (Rhoads 2009).

Arabidopsis thaliana possesses three genes encoding eIF4E (At4g18040, At1g29550, and At1g29590), one for eIF(iso)4E (At5g35620) and a further gene encoding a similar protein named novel cap-binding protein (nCBP) (At5g18110) (Ruud et al. 1998). The eIF4E and eIF(iso)4E proteins have distinctive functions. Whereas eIF4E is present in all tissues except the root specialization zones, eIF(iso)4E is particularly abundant in floral tissues and young tissue, and the proteins are

Nucleotide sequence data is available in the GenBank database under accession numbers HM131206 to HM131211.

Corresponding author: J. Walsh; E-mail: john.walsh@warwick.ac.uk

* The $e$-Xtra logo stands for "electronic extra" and indicates that a supplementary table is published online and that Figure 3 appears in color online. thought to have differing roles in plant metabolism and development (Rodriguez et al. 1998). Each is under different regulatory control pathways (Dinkova et al. 2000).

The first evidence that plant viruses interacted directly with the eukaryotic translation initiation complex of plants was provided when it was shown that the virus-encoded genomelinked protein (VPg) of Turnip mosaic virus (TuMV; genus Potyvirus), which binds to the 5' end of the viral RNA, bound to A. thaliana eIF(iso)4E in yeast two-hybrid binding assays (Wittmann et al. 1997). A recent comparison of TuMV VPg interactions with A. thaliana $\mathrm{eIF}$ (iso) $4 \mathrm{E}, \mathrm{nCBP}$, and all three eIF4E proteins in the yeast two-hybrid system revealed that only eIF(iso)4E acted as a functional partner (Gallois et al. 2010). Furthermore, in an enzyme-linked immunosorbent assay (ELISA)-based system, VPg interacted with tagged $A$. thaliana eIF(iso)4E and, to a lesser extent, with tagged $A$. thaliana eIF4E (Léonard et al. 2000).

In contrast to the in vitro results, it is the $\operatorname{eIF}$ (iso) $4 E$ gene alone that is required for successful infection of $A$. thaliana by TuMV (Sato et al. 2005). Insertional mutagenesis of At.eIF(iso) $4 E$ using a defective maize transposon tagged with an herbicide resistance gene $(\mathrm{dSpm})$ produced a plant line with no detectable mRNA or protein from the gene. This line is able to grow normally and is resistant to potyvirus infection, in particular, TuMV and Lettuce mosaic virus (Duprat et al. 2002). Additionally, an EMS-induced mutation of A. thaliana named $l s p l$ also conferred loss of susceptibility to TuMV and other members of the Potyviridae; the mutation was found to be in eIF(iso) $4 E$ (Lellis et al. 2002). In contrast, TuMV is able to infect $A$. thaliana lines with T-DNA insertions in $e I F 4 E$ (Sato et al. 2005), eIF4G, eIF(iso)4G1, or eIF(iso)4G2 (Nicaise et al. 2007).

Since these original discoveries, there have been many reports of recessive resistance to Potyvirus spp. and members of certain other virus groups (Cucumovirus, Carmovirus, and Bymovirus) resulting from mutations in eIF4E, eIF(iso) $4 E$, or the other components of the eukaryotic translation initiation complex, eIF4G or eIF(iso)4G (Robaglia and Caranta 2006). For example, the alleles pvr1/2 (Capsicum spp.) confer resistance to Potato virus $Y$ (Kang et al. 2005), rym4/5/6 (barley) to Barley yellow mosaic virus (Kanyuka et al. 2005), mol (lettuce) to Lettuce mosaic virus (Nicaise et al. 2003), sbml/wlv/cyv-2 (pea) to various viruses (Andrade et al. 2009; Bruun-Rasmussen et al. 2007; Gao et al. 2004), and bc-3 (bean) to Bean common mosaic virus (Naderpour et al. 2010), and all correspond to eIF4E. Pea sbm2, acting against Pea seed-borne mosaic virus, is linked to $\operatorname{IF}$ (iso)4E (Gao et al. 2004), Capsicum pvr6 is a null allele of pepper $e I F($ iso) $4 E$ (Ruffel et al. 2002) and retr01 in Brassica rapa has been suggested to be eIF(iso) $4 E$ 
(Rusholme et al. 2007). Mutations in $e I F 4 G$ and $e I F($ iso $4 G G$ have been identified as the resistance genes cuml and rymvl, respectively (Albar et al. 2006).

Here, we describe the identification and cloning of multiple copies of $e I F 4 E$ and $e I F(i s o) 4 E$ from $B$. rapa. Using a $d S p m$ transposon knock-out line of Arabidopsis (Duprat et al. 2002) we have been able to show, by complementation, that TuMV can utilize at least two copies of both $e I F 4 E$ and $e I F($ iso) $4 E$. This may help to explain why recessive resistance to TuMV in Brassica spp. is less common than recessive resistance to Potyvirus spp. in some other plant species.

\section{RESULTS}

Identification of $B$. rapa $e I F 4 E$ and $e I F($ iso $) 4 E$ genes.

The $B$. rapa line R-o-18 is highly inbred and uniformly susceptible to TuMV. Three copies of $e I F 4 E$ and three copies of $e I F($ iso $) 4 E$ were identified by polymerase chain reaction (PCR) on this line using primers designed to Brassica Genome Sequence Survey (GSS) and expressed sequence tag (EST) sequences. The PCR products were then used to probe a bacterial artificial chromosome (BAC) library of this line (Rana et al. 2004) to obtain the full-length genomic sequences. The structures of the genes were inferred from homology to A. thaliana genes and to $B$. napus EST sequences. Each gene consisted of five exons and four introns, with the exception of BraA.eIF4E.b, which lacked exons 2 and 3 and appeared to be a pseudogene (Fig. 1). Most of the variation between the eIF4E genes was due to size differences of intron 1 whereas the eIF(iso) $4 E$ genes differed markedly in the length of intron 3. Mapping has shown that BraA.eIF4E.a, BraA.eIF4E.c, BraA.eIF(iso)4E.a, and BraA.eIF(iso)4E.c are at different loci (Rusholme et al. 2007) (data not shown).
The sequences of the predicted proteins are shown in Figure 2. BraA.eIF(iso)4E.a and BraA.eIF(iso)4E.c were $91.0 \%$ identical to each other and 90.8 and $86.7 \%$ identical to At.eIF(iso)4E, respectively. BraA.eIF4e.a and BraA.eIF4E.c had $87.8 \%$ identity to each other but only 51.8 and $52.8 \%$ identity, respectively, to At.eIF(iso)4E.

\section{B. rapa eIF $4 E$ or $e I F$ (iso) $4 E$ genes complement an $e I F$ (iso) $4 E$ knock-out}

in A. thaliana plants for TuMV infection.

In order to determine which of the $B$. rapa genes could be used by TuMV during the infection process, copies of the genes were transformed into a Sainsbury Laboratory Arabidopsis transposant line (SLAT) possessing a nonfunctional At.eIF(iso) $4 E$ gene (Col-0::dSpm). Because TuMV is unable to infect this line (Duprat et al. 2002), any transformed lines challenged with TuMV that were susceptible would indicate functional complementation by the Brassica transgene. A. thaliana lines were transformed with BraA.eIF4E.a, BraA.eIF4E.c, BraA.eIF(iso)4E.a, and BraA.eIF(iso)4E.c. BraA.eIF4E.b was a pseudogene and BraA.eIF(iso)4E.b was not found in the BAC library; therefore, they were not included.

Col-0 plants developed severe symptoms and were stunted within 2 weeks of challenge with TuMV CDN 1, whereas Col$0:: d S p m$ plants remained symptomless, with no virus detected throughout the period of the test (Fig. 3). Infected plants were prone to secondary fungal infections and frequently rotted within 5 weeks of virus challenge. All mock- and virus-inoculated Col-0:: $d S p m$ plants remained symptomless and free of virus as determined by ELISA at 3 weeks postinoculation.

PCR tests on two to six plants per transformation confirmed the presence of wild-type At.eIF(iso) $4 E$ in Col-0 lines and the continued presence of the $d S p m$ insertion in At.eIF(iso) $4 E$ in

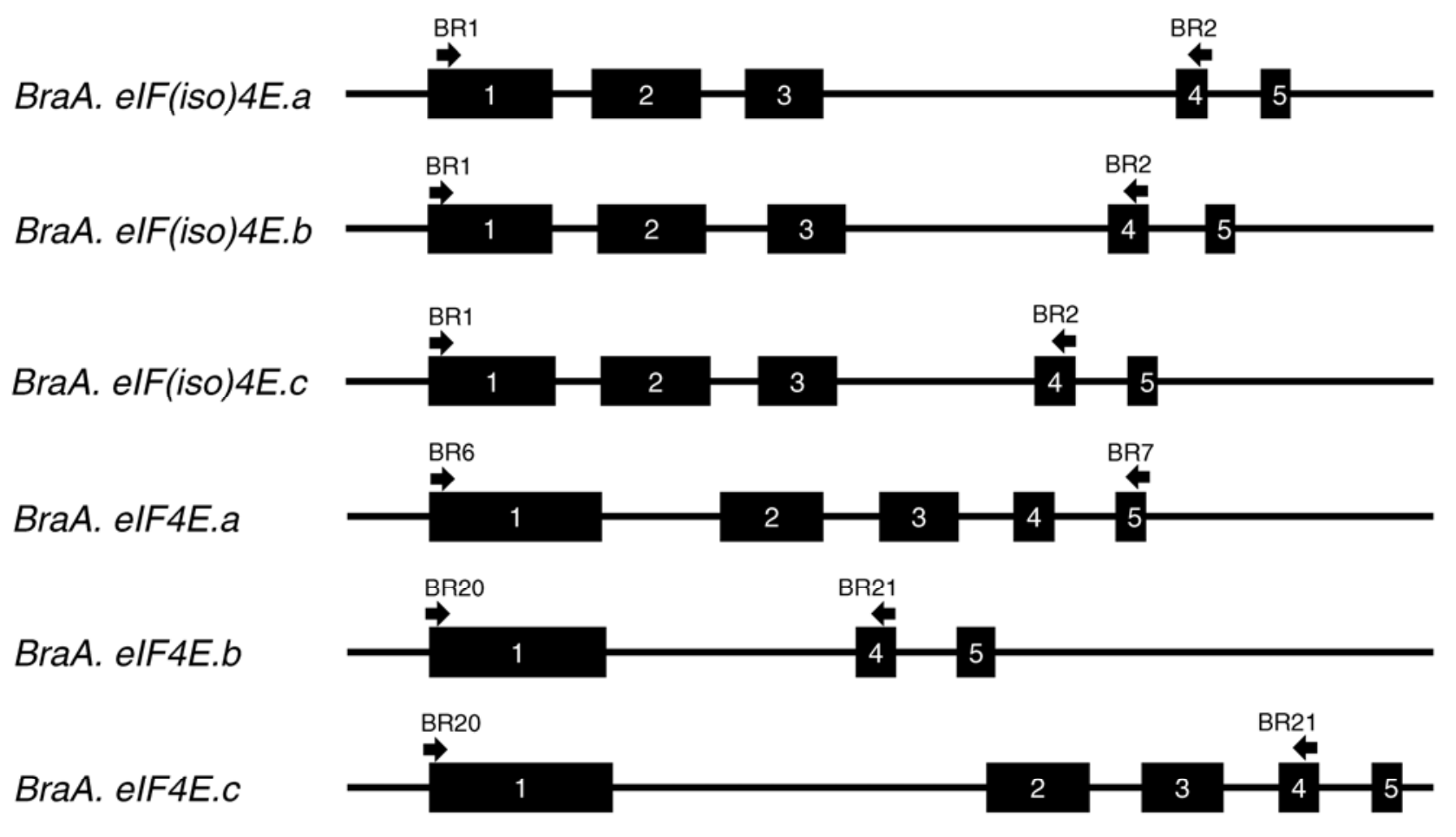

$250 \mathrm{bp}$

Fig. 1. Structure of Brassica rapa eIF $4 E$ and $e I F(i s o) 4 E$ genes indicating positions of exons, introns, and generic primers used for probe production. Exons are indicated by black boxes and introns are thin lines. Sequences were determined outside the generic primer range using BACs or genome-walking. 
all Col-0:: $d S p m$ plants tested. The $\mathrm{T}_{2}$ plants segregated for the presence of the transgenes, and the presence of the selection marker (bar or $n p t I I)$ was associated with the presence of the appropriate Brassica transgene. The absence of the selection marker corresponded with the absence of the Brassica transgene. Sequence checks confirmed there were no alterations in the transgenes.
Following TuMV challenge, plant symptoms were assessed visually and virus titers were estimated by ELISA followed by transformation for residual maximum likelihood (REML) analyses. Plants with symptoms were found to have significantly higher virus titers (ELISA optical density [OD] values) than plants with no symptoms $(P<0.001)$ (Table 1$)$. Col-0 $\mathrm{T}_{2}$ plants with Brassica transgenes appeared identical to the parental Col-
A

At.elF(iso)4E

BraA.elF(iso)4E.a BraA.elF(iso)4E.c

At.elF(iso)4E

BraA.elF(iso)4E.a

BraA.elF(iso)4E.c

At.elF(iso)4E

BraA.elF(iso)4E.a

BraA.elF(iso)4E.

B

BraA.elF4E.a BraA.elF4E.c

BraA.eIF4E.a BraA.eIF4E.c

BraA.elF4E.a BraA.eIF4E.c

10

20

30

40

50

60

70

MATDDVNEPLPAAAELPATEAEKQ- - PHKLERKUSFWFDNQS-HKKGAAWGASLRKAYTFDTVEDF WGL HETI FQT SKLT 76 MAT EDVNEALAAAEVPATETTEKO- PAHKLERKWSFWFDNQSKPKGGAAWGASLRKAYTFDTVGDF WGL HETI FIPSKLT 79

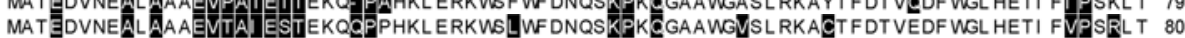

90

100

110

120

130

140

150

160

ANAEI HLFKAGVEPKWEDPECANGGKWT WWVTANRKEAL DKGWLETL MALI GEQFDEADEI CGVVASVRPQSKQDKLSLW 156 PNAEI HMFKAGVEPKWEDPECANGGKWT YVVTSNRKPAL DKAWLETLMAL VGEQFDEADEI CGVVASVRP.FKQDKLSLW 157

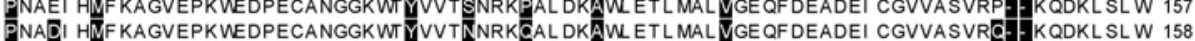

170

180

190

200

TRTKSNEAVLMGI GKKWKEI LDVTDKI TFNNHDDSRRSRFTV

TRTKSNEAVLMGI GKKWKEI LDVTDKI TFT̈NHDDSRRTRFTV

TRTKSNEAVLMGI GKKWKEI LDVTDKI SFIUNHDDARRSRFSV

20

30

40

50

60

70

80

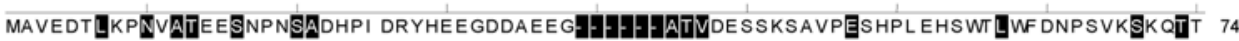
MAVEDT SKPVVVAEEANPNPTDHPI DRYHEEGDDAEEGEI AGGEGDGDESSKSAVPQSHPLEHSWT FWFDNPSVKLKQAT 80

100

110

120

130

140

150

160

WGSSLRSVFTFSTVEEFWL YNN】RHPSKLA AGADLYCFKHNI EPKWEDPI CANGGKWT MNFSREKSDKPFLYTLLALI G 154 WGSSLRSVFTFSTVEEFWSLFNNMRGPSKLAGGADFYCFKHNI EPKWEDPI CANGGKWT MNFPKEKSDKPWL YTLLALI G 160

180

190

200

210

220

230

EQFDHGDEI CGVVVNVRAKQERI SI WT KNSSNEAAQVSI GRQWKEF】DYNSSI GFI I HEDAKKLDRGAKSAYTA

EQFDHGDEI CGAVVNVRGKQERI SI WT KNASNEAAQVSI GKQWKEFI DYNNSI GFI I HEDAKKLDRGAKSAYTA

228

234

Fig. 2. Comparison of the Arabidopsis thaliana $\mathrm{IF}(\mathrm{iso}) 4 \mathrm{E}$ protein sequence with predicted proteins from Brassica rapa line R-o-18 eIF(iso) $4 E$ and $e I F 4 E$ genes. A, eIF(iso)4E proteins. Differences from the protein sequence At.eIF(iso)4E are indicated by shading. B, eIF4E proteins. Differences from the protein sequence BraA.eIF4E.c are indicated by shading.

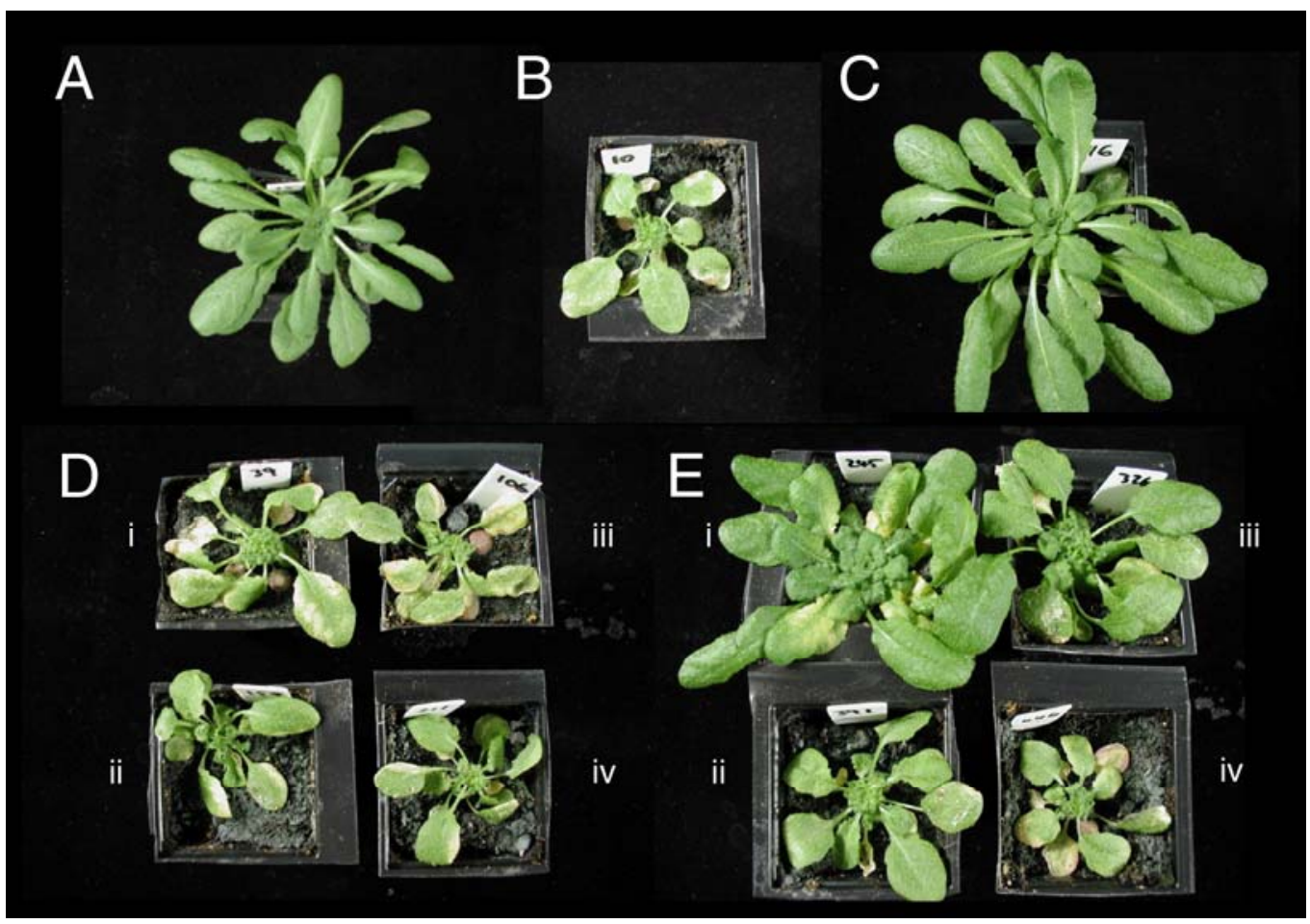

Fig. 3. Complementation of Arabidopsis thaliana plants with Brassica rapa eukaryotic translation initiation factor genes, following challenge by mechanical inoculation with Turnip mosaic virus 20 days postinoculation. A, Col-0 mock inoculated; B, Col-0; C, Col-0::dSpm; D, Col-0 with transgenes; E, Col-0:: $d S p m$ with transgenes; i, BraA.eIF4E.a; ii, BraA.eIF4E.c; iii, BraA.eIF(iso)4E.a; and iv, BraA.eIF(iso)4E.c. The dSpm transposon insertion is in At5g35620, inactivating the gene At.eIF(iso)4E. 
0 both visually (Fig. 3) and by ELISA (Table 1), indicating no effect of the transgene in this background on virus replication. Col-0:: $d S p m \mathrm{~T}_{2}$ plants lacking Brassica transgenes remained uninfected, like the parental Col-0::dSpm line. $\mathrm{T}_{2}$ Col-0::dSpm plants with Brassica transgenes produced symptoms as severe as the Col-0 line, with the exception of Col-0::dSpm transformed with BraA.eIF4E.a, where plants had notably milder symptoms. There were significant $(P<0.001)$ differences in the amount of virus detected between lines with symptoms (Table 1, occasion no. 1), although limited numbers of plants were analyzed (both as individual families and by pooling families per transgene). Only Col-0:: $d S p m$ families transformed with BraA.eIF4E.a had a significantly $(P<0.05)$ lower virus titer than infected Col-0 plants. When larger numbers of plants from one family per transgene were tested (Table 1, occasion no. 2), results confirmed that the mild symptoms seen on Col-0:: $d S p m$ plants with the BraA.eIF4E.a transgene related to a significantly $(P<0.05)$ lower virus titer than the other infected lines.

Plants challenged with TuMV using aphids were less efficiently infected (all potentially susceptible controls were not infected), and produced milder symptoms than those challenged mechanically. The virus titer as measured by ELISA was also significantly reduced $(P<0.001)$ (Table 2$)$. The lower levels of symptom severity and virus titer achieved by aphid transmission are likely to reflect a lack of TuMV acquisition by aphids or a smaller viral inoculum load.

The symptoms were remarkable in the consistency of visual severity between plants within any one $\mathrm{T}_{2}$ family (derived from a $\mathrm{T}_{1}$ plant with a Brassica transgene); plants appeared either stunted to equal degrees or totally healthy. There was also consistency in visual symptom severity between plants of different families containing the same Brassica transgene. If the $T_{1}$ plants had possessed a single copy of the transgene, the $T_{2}$ families would be expected to segregate $3: 1$ for the presence of the transgene, with a quarter of plants having two copies of the transgene, half having one copy (heterozygotes), and one quarter having no copies. The consistency of infection levels (visual or ELISA) suggested that homozygous and heterozygous transgenic plants could not be distinguished on the basis of degree of virus susceptibility.

\section{DISCUSSION}

It has been shown that very few Potyvirus spp. use eIF4E in one host and eIF(iso)4E in another host for protein translation; for example, Tobacco etch virus (TEV) uses eIF4E in pepper (Kang et al. 2005) and tomato (Ruffel et al. 2005) but eIF(iso)4E in A. thaliana (Lellis et al. 2002). Also, it is very rare that a Potyvirus sp. uses both eIF4E and eIF(iso)4E in the same host; for example, Pepper veinal mottle virus (PVMV) uses both in pepper (Ruffel et al. 2006). Because TuMV infection of $A$. thaliana requires functional eIF(iso)4E (Duprat et al. 2002; Lellis et al. 2002), we asked whether the same preference would be shown for genes from B. rapa, an economically important host. Additionally, because $B$. rapa possesses multiple loci of $e I F 4 E$ and $e I F$ (iso) $4 E$, we investigated the ability of TuMV to use the genes at the different loci. A requirement for a particular gene would be an excellent target for the basis of recessive resistance, either natural or engineered via gene silencing (Zhang et al. 2006). Alternatively, it would be impor-

Table 1. Phenotypes of transgenic Arabidopsis thaliana plants possessing Brassica rapa eukaryotic translation initiation factor genes following mechanical inoculation with Turnip mosaic virus (visual symptoms and enzyme-linked immunosorbent assay [ELISA]) ${ }^{\mathrm{a}}$

\begin{tabular}{|c|c|c|c|c|c|c|c|c|c|}
\hline \multirow[b]{3}{*}{ Brassica transgene } & \multirow[b]{3}{*}{$\mathbf{T}_{2}$ family } & \multicolumn{4}{|c|}{ No. of plants with symptoms ${ }^{b}$} & \multicolumn{4}{|c|}{ Mean ELISA optical density (no. of plants) ${ }^{c}$} \\
\hline & & \multicolumn{2}{|c|}{ Col-0 } & \multicolumn{2}{|c|}{ Col-0::dSpm } & \multicolumn{2}{|c|}{ Col-0 } & \multicolumn{2}{|c|}{ Col-0::dSpm } \\
\hline & & $\mathbf{0}$ & $\mathbf{S M}$ & $\mathbf{0}$ & SM & $\mathbf{0}$ & $\mathbf{S M}$ & $\mathbf{0}$ & $\mathbf{S M}$ \\
\hline \multicolumn{10}{|l|}{ Occasion no. 1} \\
\hline None & $\ldots$ & 0 & 12 & 12 & 0 & - & $1.77(3)$ & $-0.03(12)$ & - \\
\hline \multirow[t]{5}{*}{ BraA.eIF4E.a } & $\mathrm{i}$ & 0 & 12 & 4 & 8 & - & $1.58(3)$ & $0.00(2)$ & $0.33(2)$ \\
\hline & ii & 0 & 12 & 1 & 11 & - & $1.65(3)$ & $0.01(1)$ & $0.28(3)$ \\
\hline & iii & 0 & 12 & 4 & 8 & - & $1.74(3)$ & $0.00(2)$ & $0.40(3)$ \\
\hline & iv & 0 & 12 & 7 & 5 & - & $1.73(3)$ & $0.02(2)$ & $0.28(2)$ \\
\hline & Total & 0 & 48 & 16 & 32 & - & $1.68(12)$ & $0.01 * * *(7)$ & $0.32 *(10)$ \\
\hline \multirow[t]{5}{*}{ BraA.eIF4E.c } & $\mathrm{i}$ & 0 & 12 & 5 & 7 & - & $1.64(3)$ & $0.02(1)$ & $1.13(3)$ \\
\hline & ii & 0 & 12 & 2 & 10 & - & $1.57(3)$ & $0.03(1)$ & $1.21(3)$ \\
\hline & iii & 0 & 12 & 2 & 10 & - & $1.30(3)$ & $0.00(1)$ & $0.75(3)$ \\
\hline & iv & 0 & 12 & 1 & 11 & - & $1.70(3)$ & $0.03(1)$ & $0.62(3)$ \\
\hline & Total & 0 & 48 & 10 & 38 & - & $1.55(12)$ & $0.02 * * *(4)$ & $0.93(12)$ \\
\hline \multirow[t]{5}{*}{ BraA.eIF(iso)4E.a } & $\mathrm{i}$ & 0 & 12 & 2 & 10 & - & $1.54(3)$ & 0.04 (1) & $1.53(3)$ \\
\hline & ii & 0 & 12 & 0 & 12 & - & $1.60(3)$ & - & $0.53(4)$ \\
\hline & iii & 0 & 12 & 3 & 9 & - & $1.31(3)$ & $0.01(1)$ & $1.01(3)$ \\
\hline & iv & 0 & 12 & 3 & 9 & - & $1.40(3)$ & $0.01(1)$ & $0.81(3)$ \\
\hline & Total & 0 & 48 & 8 & 40 & - & $1.50(12)$ & $0.02 * * *(3)$ & $0.94(13)$ \\
\hline \multirow[t]{5}{*}{ BraA.eIF(iso)4E.c } & $\mathrm{i}$ & 0 & 12 & 7 & 5 & - & $1.70(3)$ & $0.01(2)$ & $0.25(2)$ \\
\hline & ii & 0 & 12 & 5 & 7 & - & $1.60(3)$ & $0.02(2)$ & $1.15(2)$ \\
\hline & iii & - & - & 0 & 12 & - & - & - & $0.58(4)$ \\
\hline & iv & - & - & 1 & 11 & - & - & $0.02(1)$ & $0.92(3)$ \\
\hline & Total & 0 & 24 & 13 & 25 & & $1.65(6)$ & $0.02 * * *(5)$ & $0.77(11)$ \\
\hline \multicolumn{10}{|l|}{ Occasion no. 2} \\
\hline None & $\ldots$ & 0 & 12 & 12 & 0 & - & $1.14(12)$ & $0.00 * * *(12)$ & - \\
\hline BraA.eIF4E.a & $\mathrm{i}$ & 0 & 12 & 6 & 6 & - & 1.18 (12) & $-0.01 * * *(6)$ & $0.17 *(6)$ \\
\hline BraA.eIF4E.c & $\mathrm{i}$ & 0 & 12 & 3 & 9 & - & $1.17(12)$ & $-0.01 * * *(3)$ & $1.23(9)$ \\
\hline BraA.eIF(iso)4E.a & $\mathrm{i}$ & 0 & 12 & 0 & 12 & - & $1.20(12)$ & - & $1.21(12)$ \\
\hline BraA.eIF(iso)4E.c & $\mathrm{i}$ & 0 & 12 & 11 & 1 & - & $1.26(12)$ & $-0.03 * * *(11)$ & $1.41(1)$ \\
\hline
\end{tabular}

\footnotetext{
a $d S p m$ transposon insertion is in At5g35620, inactivating the gene At.eIF(iso)4E. Symptoms observed: $0=$ no symptoms, SM = systemic mosaic with stunting and leaf deformation, $-=$ not applicable.

b Twelve plants inoculated per $\mathrm{T}_{2}$ family. Families have not been selected for transgene homozygosity and are segregating for the presence of the transgene.

${ }^{\mathrm{c}}$ ELISA optical density measured at $405 \mathrm{~nm}$ after $2 \mathrm{~h}$. Statistically significant differences (by residual maximum likelihood) compared with Col-0 plants with no transgene are indicated by $*$ and $* * *(P<0.05$ and 0.001 , respectively $)$.
} 
tant to know whether TuMV is able to use different copies of $e I F 4 E$ or $e I F$ (iso) $4 E$ or whether it has a requirement for more than one copy, in order to deploy such resistance in B. rapa.

Our results are unusual in that TuMV exhibits the ability to use four different translation initiation factor proteins to counteract the absence of At.eIF(iso)4E in the knock-out $A$. thaliana line. This is the first example of a virus being able to use different copies of $e I F 4 E$ and $e I F(i s o) 4 E$ from multiple loci of the same plant. The use of simultaneous knock-outs of At.eIF(iso) 4G1 and At.eIF(iso)4G2 has been reported to prevent TuMV infection of $A$. thaliana, whereas plants with only one of the two genes inactivated are susceptible (Nicaise et al. 2007). However, the ability to use four isoforms (two each of BraA.eIF4E and BraA.eIF(iso)4E) from one host species is remarkable. A slightly similar situation occurs for Chilli veinal mottle virus (ChiVMV), whose VPg interacts (in yeast two-hybrid studies) with both Capsicum eIF4E and eIF(iso)4E (Hwang et al. 2009). Mutations or gene silencing of both these genes together (also known as $p v r 1 / 2$ and $p v r 6$ ) confers resistance to ChiVMV and PVMV (Ruffel et al. 2006), although it is unclear whether Capsicum annuum possesses other copies of either gene.

Of the four translation initiation factor genes, TuMV replication was least supported by BraA.eIF4E. $a$ when expressed in A. thaliana. Further data would be needed to understand this; for example, whether the underlying cause is due to different levels of transcription or translation (and whether this is also the case in $B$. rapa) or due to protein structure. However, comparison of the protein sequences with the resolved structure of wheat eIF4E (Monzingo et al. 2007) revealed that the four Brassica proteins possess all the conserved residues noted as important in capbinding and for stabilizing the structure of the protein, with the notable exception of a key tryptophan residue lacking in BraA.eIF4E.a (residue 145, replaced by phenylalanine).

It is likely that the viral protein interacting with the $B$. rapa eIF4E/eIF(iso)4E proteins is the VPg. A single amino acid substitution in TuMV VPg that can abolish the interaction with Arabidopsis eIF(iso)4E in yeast two-hybrid experiments simultaneously renders the virus unable to infect $B$. perviridis (a synonym for $B$. rapa var. perviridis) (Léonard et al. 2000). TuMV VPg has been reported to interact with one version of eIF(iso)4E from $B$. rapa in the yeast two-hybrid system (Hwang et al. 2009), although the authors did not describe different versions of the gene. Co-purification studies from $B$. perviridis have confirmed that TuMV VPg (as a precursor protein with or without the adjacent $6 \mathrm{~K}$ protein and Pro protease sections) does interact in planta with eIF(iso)4E/eIF4E (Léonard et al. 2004). Infection by TuMV alters the profile of isomers found; Léonard and associates (2004) reported finding eIF(iso)4E in mock-inoculated and TuMV-infected plants but detectable levels of eIF4E in infected plants only. The two proteins appear to have different subcellular locations in Nico- tiana benthamiana, eIF(iso)4E being primarily associated with rough endoplasmic reticulum and eIF4E with free ribosomes (Beauchemin et al. 2007). Because different forms of VPg are found in different cellular locations, Beauchemin and associates (2007) suggested that, in $N$. benthamiana at least, the $6 \mathrm{~K}$ VPg-Pro is the most likely form to be involved in viral translation (with eIF(iso)4E) and the VPg-Pro form may be involved in other cellular perturbations. TuMV VPg can bind to, but does not disrupt, the eIF(iso)4E-eIF(iso)4G complex, and it is believed to compete with capped cellular mRNAs for the translation machinery (Miyoshi et al. 2006; Plante et al. 2004). However, it should be noted that other potyviral proteins have associations with eIF4E, such as NIa interacting with eIF4E in yeast two-hybrid tests (Schaad et al. 2000) or CI being involved in eIF4E-based resistance breaking (Abdul-Razzak et al. 2009).

Kang and associates (2007) found that transforming tomato with $e I F 4 E$ from the $p v r l$ locus in pepper resulted in dominant resistance to several TEV strains and other Potyvirus spp. In our experiments, wild-type Col-0 A. thaliana plants transformed with $e I F 4 E$, or $e I F$ (iso) $4 E$ from $B$. rapa, were as susceptible to TuMV as untransformed wild-type plants.

Of the known plant virus resistances where heritability has been characterized, recessive resistances are more common for Potyvirus spp. than for viruses of other families; $64 \%$ of the examples listed by Díaz-Pendón and associates (2004) corresponded to Potyvirus spp. In their review of sources of resistance to Potyvirus spp., Provvidenti and Hampton (1992) showed that, where heritability had been determined, 60 genes were dominant and 39 were recessive. They listed two examples of recessive resistance, compared with three examples of dominant resistance in Brassica spp. A review of resistances to TuMV listed four dominant and three recessive resistances (Shattuck 1992). Despite a large amount of screening over a prolonged period, we have found that recessive resistances to TuMV are relatively scarce in Brassica spp.; we have mapped six dominant resistance genes and one recessive gene (Walsh and Jenner 2002) and it took a screen of more than 3,000 lines of the diploid $B$. rapa (Liu et al. 1996) to find the latter. This recessive resistance was effective against a range of TuMV isolates from different parts of the world and representing different serotypes, pathotypes, and genetic groups (Walsh et al. 2002) and, thus far, has not been broken. Two loci controlling the resistance were mapped; the first gene, retr01, was recessive and the second, ConTR01, was dominant (Rusholme et al. 2007). We also showed that the A genome of B. napus (derived from B. rapa) appears to have three $e I F 4 E$ loci and three $e I F$ (iso) $4 E$ loci and that retrO1 appeared to be coincident with one of the $e I F$ (iso) $4 E$ loci and ConTRO1 appeared to be coincident with one of the loci of $e I F 4 E$, or possibly one of the other loci of eIF(iso) $4 E$ (Rusholme et al. 2007). A possible explanation for the relative scarcity of recessive resistances to TuMV in Brassica spp. is

Table 2. Comparison of mechanical and aphid inoculation on degree of infection by Turnip mosaic virus of transgenic Arabidopsis thaliana plants possessing Brassica rapa eukaryotic translation initiation factor $e I F($ iso $) 4 E a^{a}$

\begin{tabular}{|c|c|c|c|c|c|c|c|c|c|}
\hline \multirow[b]{3}{*}{ Brassica transgene } & \multirow[b]{3}{*}{ Inoculation method } & \multicolumn{4}{|c|}{ No. of plants with symptoms } & \multicolumn{4}{|c|}{ Mean ELISA optical density } \\
\hline & & \multicolumn{2}{|c|}{ Col-0 } & \multicolumn{2}{|c|}{ Col-0::dSpm } & \multicolumn{2}{|c|}{ Col-0 } & \multicolumn{2}{|c|}{ Col-0::dSpm } \\
\hline & & $\mathbf{0}$ & SM & $\mathbf{0}$ & SM & $\mathbf{0}$ & SM & $\mathbf{0}$ & SM \\
\hline \multirow[t]{2}{*}{$\overline{\text { None }}$} & Mechanical & 0 & 5 & 5 & 0 & - & 2.67 & $0.15 * * *$ & - \\
\hline & Aphid & 2 & 3 & 5 & 0 & $0.05 * * *$ & $0.52 * * *$ & $0.04 * * *$ & - \\
\hline \multirow[t]{2}{*}{ BraA.eIF(iso)4E.a } & Mechanical & 0 & 12 & 4 & 8 & - & 2.72 & $0.11 * * *$ & 2.58 \\
\hline & Aphid & 6 & 6 & 7 & 5 & - & $0.48 * * *$ & $0.04 * * *$ & $0.41 * * *$ \\
\hline
\end{tabular}

a dSpm transposon insertion is in At5g35620, inactivating the gene At.eIF(iso)4E. Symptoms observed: $0=$ no symptoms, SM = systemic mosaic with stunting and leaf deformation, and $-=$ not applicable.

${ }^{\mathrm{b}}$ Twelve plants inoculated per $\mathrm{T}_{2}$ family. Families have not been selected for transgene homozygosity and are segregating for the presence of the transgene.

${ }^{\mathrm{c}}$ Enzyme-linked immunosorbent assay (ELISA) optical density measured at $405 \mathrm{~nm}$ after $1.5 \mathrm{~h}$, all plants were tested. Statistically significant differences (by residual maximum likelihood) compared with Col-0 mechanically-inoculated plants are indicated by $* * *(P<0.001)$. 
that, for a plant to be resistant, all copies of eIF4E and $e I F$ (iso) $4 E$ would have to be nonfunctional for TuMV. In amphidiploid Brassica spp. (B. napus, B. juncea, and B. carinata), recessive resistance based on $e I F 4 E$ or $e I F(i s o) 4 E$ is potentially less likely, because there appear to be six loci of each gene present (three of each in the A genome and three of each in the $\mathrm{C}$ genome) (Rusholme et al. 2007).

\section{MATERIALS AND METHODS}

\section{TuMV.}

TuMV CDN 1 is a pathotype 4 isolate (Walsh 1989) able to overcome several dominant Brassica resistance genes. It was maintained in $B$. juncea $\mathrm{cv}$. Tendergreen by mechanical inoculation as previously described (Walsh 1989). Aphid transmission was performed using Myzus persicae cultures raised on $B$. napus cv. Mikado. Aphids were starved for approximately $2 \mathrm{~h}$, then allowed to feed on infected $B$. juncea leaves for a few minutes before they were transferred onto test plants (five per plant) for 2 days and, finally, killed using Aphox (Syngenta, Cambridge) insecticide.

\section{Plant lines.}

B. rapa subsp. trilocularis (yellow sarson) line R-o-18 is an inbred line highly susceptible to TuMV (Hughes et al. 2002). A BAC library of genomic DNA of R-o-18 (JBr, using vector pBIBAC2, average insert size $128 \mathrm{~kb}$ ) was made by I. Bancroft, John Innes Centre (Norwich, U.K.) (Rana et al. 2004).

A. thaliana Col-0 is highly susceptible to TuMV infection. Col-0:: $d S p m$ is a SLAT line that is homozygous for a transposon $(\mathrm{dSpm})$ insertion in exon 2 of At5g35620 (At.eIF(iso)4E) (Duprat et al. 2002). The transposon carries resistance to the herbicide glufosinate ammonium. The presence and location of the transposon was verified using the primer pairs dspm14E2K01 and dspm2-4E2KO4 as described previously (Duprat et al. 2002). Col-0::dSpm is resistant to infection by TuMV CDN 1.

\section{Identification of full-length $\boldsymbol{e I F}$ gene sequences from $B$. rapa.}

Genomic DNA was extracted from young R-o-18 leaves using a DNeasy plant mini kit (Qiagen, Crawley, U.K.) followed by amplification using the GenomiPhi system (GE Healthcare, Little Chalfont, U.K.), both processes performed according to the manufacturers' recommendations.

Generic primers were designed using GSS and EST sequences with homology to known $e I F 4 E$ and $e I F$ (iso) $4 E$ genes to encompass the majority of the open reading frame (ORF) (Table 3). The sequences used for eIF4E were from $B$. oleracea (BH540903, BZ068573, and BZ449347), B. rapa (CX270207,
CX270456, and DY010188), and B. napus (CB686150, CD834916, CN733851, CX188254, CX189337, CX194455, CX195737, and CX280257); for $e I F$ (iso) $4 E$, sequences were from B. oleracea (BZ436642, BZ514129, EH413959, EH414327, EH425132, EH426021, and EH426034), B. rapa (CX266566 and CX268848), and B. napus (D. Lydiate and E. Higgins, personal communication).

PCR was performed on genomic DNA using standard protocols with Taq DNA polymerase (Invitrogen, Paisley, U.K.). Primer pair BR1-BR2 produced multiple products of differing sizes from which could be separated BraA.eIF(iso)4E.a $(1,215$ bp), BraA.eIF(iso)4E.b (1,123 bp), and BraA.eIF(iso)4E.c (995 bp). Primer pairs BR6-BR7 and BR20-BR21 amplified most of the coding region of BraA.eIF4E.a (1,158 bp) and BraA.eIF4E.c (1,435 bp), respectively. PCR products were cloned into pCR2.1 (Invitrogen) or pMOSBlue (GE Healthcare) prior to sequencing at least three colonies with the BigDye Terminator system (Applied Biosystems, Warrington, U.K.) with products run on an ABI Prism 3130xl Genetics Analyzer (Applied Biosystems).

The PCR products were labeled with ${ }^{32} \mathrm{P}$ dCTP using the RediPrimeII DNA labeling system (GE Healthcare) and standard protocols. The radiolabeled probes were hybridized to filters printed with 36,864 colonies of the JBr BAC library. Potential bacterial clones of interest were identified, purchased, and subjected to a further PCR using the original primers to verify the genes' presence. Between one and three BAC per gene were thus identified (e.g., BraA.eIF4E.a in JBr034F07, BraA.eIF4E.c in JBr039L10, BraA.eIF(iso)4E.a in JBr043O19, and BraA.eIF(iso)4E.c in JBr040N12), although no BAC with BraA.eIF(iso)4E.b was found. Two BAC (JBr042K14 and $\mathrm{JBr} 043 \mathrm{~K} 20$ ) were found to contain an $e I F 4 E$-like gene that lacked exons 2 and 3 and had stop codons in the normal reading frame of exons 1 and 4 . This defective gene was designated BraA.eIF4E.b.

Primers matching the PCR product sequences were designed to extend the known sequence into the BAC DNA. Sequence was determined upstream and downstream of the genes in a stepwise manner for approximately $2 \mathrm{~kb}$ in each direction beyond the start and stop codons of the ORF (Supplementary Table 1).

\section{Construction of binary vectors.}

Having identified the start and stop codons of the ORF, primers BR57 to BR64 (Table 3) were used to amplify the Brassica genes together with likely promoters (200 to $600 \mathrm{bp}$ upstream of the start codon). In the case of BraA.eIF4E.a, the upstream primer location was restricted by the need to avoid an upstream ORF discovered in close proximity. Downstream regions were chosen to include likely sites of polyA tail attach-

Table 3. Primers used for the identification and cloning of Brassica rapa eIF4E and $e I F($ iso $) 4 E$ genes

\begin{tabular}{|c|c|c|c|c|}
\hline Primer & Sequence $\left(5^{\prime}-3^{\prime}\right)$ & Genes & Location & Direction \\
\hline BR1 & ATGGCGACAGAGGATGTGAACG & BraA.eIF(iso)4E.a, $b, c$ & Exon 1 & Forward \\
\hline BR2 & ТСТССТТССАСТТСТТСССААТАС & BraA.eIF(iso)4E.a, $b, c$ & Exon 4 & Reverse \\
\hline BR6 & CACTCTCAAGCCTAATGTCGC & BraA.eIF4E.a & Exon 1 & Forward \\
\hline BR7 & AGAGGTTGTAGGTTTCAGGCAGTG & BraA.eIF4E.a & Exon 5 & Reverse \\
\hline BR20 & CAATGGCGGTAGAAGACACTT & BraA.eIF4E.b, $c$ & Exon 1 & Forward \\
\hline BR21 & CATGGATGATGAAACCAATGT & BraA.eIF4E.b, $c$ & Exon 4 & Reverse \\
\hline BR57 & AAAAAGCAGGCTTTTGGTCTGCAGTTATGTTATTAG & BraA.eIF4E.a & Upstream & Forward \\
\hline BR58 & AGAAAGCTGGGTAAAAAGGCTTGCGAGTCA & BraA.eIF4E.a & Downstream & Reverse \\
\hline BR59 & AAAAAGCAGGCTTAGGACAAATGATATGGGGAGAGT & BraA.eIF4E.c & Upstream & Forward \\
\hline BR60 & AGAAAGCTGGGTAGCTTGGCGACCTTTTGA & BraA.eIF4E.c & Downstream & Reverse \\
\hline BR61 & AAAAAGCAGGCTACCCTTAGTATCAATTAATTCAGAAC & BraA.eIF(iso)4E.a & Upstream & Forward \\
\hline BR62 & AGAAAGCTGGGTTCCTGGTCGAAATCAATAAGA & BraA.eIF(iso)4E.a & Downstream & Reverse \\
\hline BR63 & AAAAAGCAGGCTTTTTTAAGAATGGAGGGAGTAT & BraA.eIF(iso)4E.c & Upstream & Forward \\
\hline BR64 & AGAAAGCTGGGTGAAGCGCGGGTCAAAAT & BraA.eIF(iso)4E.c & Downstream & Reverse \\
\hline
\end{tabular}


ment as found in comparable Brassica EST. The sizes of the cloned regions were BraA.eIF4E.a, 2,144 bp; BraA.eIF4E.c, 2,758 bp; BraA.eIF(iso)4E.a, 2,076 bp; and BraA.eIF(iso)4E.c, $1,818 \mathrm{bp}$

The genes were amplified from the BAC using KOD Hotstart DNA polymerase (Merck, Nottingham, U.K.), and attB adapters were added by PCR with the same enzyme and Gateway primers attB1 and attB2 (Invitrogen). The products were cloned into the Gateway Entry plasmid pDONR221 using BP clonase (Invitrogen).

Binary vectors pB2GW7 and pK2GW7 (Karimi et al. 2002) contain a $35 \mathrm{~S}$ constitutive promoter, a $35 \mathrm{~S}$ terminator, and glufosinate ammonium herbicide resistance (bar) or kanamycin resistance (nptII), respectively, for in planta selection. The Brassica genes were transferred from the Entry vector into the binary vectors using an LR clonase-mediated reaction (Invitrogen). Sequences were verified at all stages of the cloning.

\section{Transformation of $B$. rapa genes into Arabidopsis thaliana.}

Purified plasmid DNA of each construct was electroporated into Agrobacterium tumefaciens GV3101 pMP90 using standard techniques. The presence and stability of the transgenes was verified by PCR.

Arabidopsis thaliana Col-0 and Col-0::dSpm lines were grown and transformed with Agrobacterium tumefaciens containing the constructs using the floral dip method (Clough and Bent 1998). Constructs in pB2GW7 were used to transform Col-0; pK2GW7 was used to transform Col-0::dSpm. Each Brassica gene was transformed into each plant line (Table 4).

The primary transformed plants were allowed to set seed and the $T_{1}$ seed was harvested. For selection using glufosinate ammonium, seed was grown in trays on F2S seed compost (Levington, Suffolk, U.K.) that was drenched every 3 to 4 days with glufosinate ammonium (Harvest; Bayer Crop Science, Cambridge) at $150 \mathrm{mg} / \mathrm{liter}$ for 3 weeks. For selection using kanamycin, seed was grown in deep petri dishes on $0.5 \times$ Murashige and Skoog medium (Duchefa Biochemie, Melford Laboratories, Ipswich, U.K.) with kanamycin (Sigma-Aldrich, Gillingham, U.K.) at $50 \mu \mathrm{g} / \mathrm{ml}$ for 3 weeks. Genomic DNA from individual $\mathrm{T}_{1}$ survivors was obtained as above and PCR was performed to check for the presence of the transgenes (Brassica genes and selection markers) prior to potting on the plants in compost without selection to allow the plants to flower and to set seed $\left(\mathrm{T}_{2}\right)$.

$\mathrm{T}_{2}$ plants were grown and genomic DNA was extracted as above. PCR checks were performed using the Brassica primers used to generate the inserts for the binary clones with KOD Hotstart DNA polymerase. The products were sequenced to confirm that there were no errors in the final transgenes. The continued presence of $d S p m$ in At.eIF(iso) $4 E$ was also checked in appropriate lines.

\section{TuMV challenge of $A$. thaliana lines transformed with $B$. rapa genes.}

For each transformed line, seed of up to four $\mathrm{T}_{2}$ families (-1 to -4 ), derived from different $\mathrm{T}_{2}$ individuals, were sown. Only

Table 4. Designations of lines of Arabidopsis thaliana transformed with Brassica rapa genes

\begin{tabular}{lcc}
\hline & \multicolumn{2}{c}{ A. thaliana } \\
\cline { 2 - 3 } Brassica gene & Col-0 & Col-0::dSpm $^{\mathbf{a}}$ \\
\hline BraA.eIF4E.a & TP085 & AT25 \\
BraA.eIF4E.c & TP082 & AT22 \\
BraA.eIF(iso)4E.a & TP083 & AT23 \\
BraA.eIF(iso)4E.c & TP084 & AT24 \\
\hline
\end{tabular}

${ }^{a}$ Possesses $d S p m$ transposon insertion in At5g35620, inactivating the gene At.eIF(iso) 4 E. two families were produced from the transformation of Col-0 with BraA.eIF(iso)4E.c. Fifteen plants of each family were sown in F2S compost and grown at $20^{\circ} \mathrm{C}$ with a 9-h day length to maintain the vegetative state. After 33 days, four to six true leaves of 12 plants were mechanically inoculated using TuMVinfected $B$. juncea leaf sap and a cotton bud to hold the inoculum. The remaining plants were inoculated with buffer only.

Symptoms were assessed visually each week for 4 weeks. At 3 weeks postinoculation, leaf samples from two plants were collected for genomic DNA extraction. The presence of Brassica transgenes, selection marker genes, and the $d S p m$ transposon in At.eIF(iso) $4 E$ were all checked by PCR. TuMV presence in three other plants was assayed by plate-trapped antigen ELISA using the mouse monoclonal antibody EMA67, essentially as previously described (Jenner et al. 1999), in order to confirm that symptoms were due to viral infection and not transgene presence. Leaf sap was extracted and diluted with $1 \mathrm{ml}$ of water, then diluted one-third before loading on microtiter plates. OD values were measured after $2 \mathrm{~h}$, or earlier if approaching the maximum measureable by the ELISA plate reader. Because infection of all the families containing the transgenes in the first experiment was unexpected, determination of virus titers in every individual plant was technically difficult. Therefore, the experiment was repeated for each of the four transgenes using mechanical inoculation, examining every individual plant of a single family per transgene (family i: TP085, TP082, TP083, TP084, AT25, АT22, АT23, and AT24) in order to determine the virus titer. A further experiment examined one family of lines possessing the BraA.eIF(iso)4E.a transgene (family iv: TP083 and AT23) in order to compare mechanical inoculation with aphid inoculation directly.

Due to the nature of the design of the experiments, data were analyzed using REML. REML allows estimation of variance components and treatment effects of a linear model with both fixed and random effects. It can be used, as in this case, to analyze unbalanced data sets. Data were transformed according to $\log _{\mathrm{e}}(100 \times[$ mean OD +0.05$])$, then analyzed for each experimental occasion.

\section{ACKNOWLEDGMENTS}

The $\mathrm{JBr}$ library used was funded by the Biotechnology and Biological Sciences Research Council (BBSRC) Brassica IGF programme. The work was supported by a BBSRC Crop Science Initiative grant (BB/E00668X/1) to J. A. Walsh. We thank D. Lydiate and E. Higgins for providing B. napus sequence information, C. Robaglia for the kind donation of the Arabidopsis dSpm line, R. Edwards for sequencing support, and J. Jones for statistical analyses.

\section{LITERATURE CITED}

Abdul-Razzak, A., Guiraud, T., Peypelut, M., Walter, J., Houvenaghel, M.C., Candresse, T., Le Gall, O., and German-Retana, S. 2009. Involvement of the cylindrical inclusion $(\mathrm{CI})$ protein in the overcoming of an eIF4E-mediated resistance against Lettuce mosaic potyvirus. Mol. Plant Pathol. 10:109-113.

Albar, L., Bangratz-Reyser, M., Hébrard, E., Ndjiondjop, M.-N., Jones, M., and Ghesquière, A. 2006. Mutations in the eIF(iso)4G translation initiation factor confer high resistance of rice to Rice yellow mottle virus. Plant J. 47:417-426.

Allen, M. L., Metz, A. M., Timmer, R. T., Rhoads, R. E., and Browning, K. S. 1992. Isolation and sequence of the cDNAs encoding the subunits of the isozyme form of wheat-protein synthesis initiation factor-4F. J. Biol. Chem. 267:23232-23236.

Andrade, M., Abe, Y., Nakahara, K. S., and Uyeda, I. 2009. The cyv-2 resistance to Clover yellow vein virus in pea is controlled by the eukaryotic initiation factor 4E. J. Gen. Plant Pathol. 75:241-249.

Beauchemin, C., Boutet, N., and Laliberté, J.-F. 2007. Visualization of the interaction between the precursors of $\mathrm{VPg}$, the viral protein linked to the genome of Turnip mosaic virus, and the translation eukaryotic initiation factor iso 4E in planta. J. Virol. 81:775-782. 
Browning, K. S. 1996. The plant translational apparatus. Plant Mol. Biol. 32:107-144

Bruun-Rasmussen, M., Møller, I. S., Tulinius, G., Hansen, J. K. R., Lund, O. S., and Johansen, I. E. 2007. The same allele of translation initiation factor 4E mediates resistance against two Potyvirus spp. in Pisum sativum. Mol. Plant-Microbe Interact. 20:1075-1082.

Clough, S. J., and Bent, A. F. 1998. Floral dip: A simplified method for Agrobacterium-mediated transformation of Arabidopsis thaliana. Plant J. 16:735-743.

Díaz-Pendón, J. A., Truniger, V., Nieto, C., García-Mas, J., Bendahmane, A., and Aranda, M. A. 2004. Advances in understanding recessive resistance to plant viruses. Mol. Plant Pathol. 5:223-233.

Dinkova, T. D., Aguilar, R., and Sánchez De Jiménez, E. 2000. Expression of maize eukaryotic initiation factor (eIF) iso4E is regulated at the translational level. Biochem. J. 351:825-831.

Duprat, A., Caranta, C., Revers, F., Menand, B., Browning, K. S., and Robaglia, C. 2002. The Arabidopsis eukaryotic initiation factor (iso)4E is dispensable for plant growth but required for susceptibility to potyviruses. Plant J. 32:927-934.

Gallois, J.-L., Charron, C., Sánchez, F., Pagny, G., Houvenaghel, M.-C., Moretti, A., Ponz, F., Revers, F., Caranta, C., and German-Retana, S. 2010. Single amino acid changes in the Turnip mosaic virus viral genome-linked protein (VPg) confer virulence towards Arabidopsis thaliana mutants knocked out for eukaryotic initiation factors eIF(iso)4E and eIF(iso)4G. J. Gen. Virol. 91:288-293.

Gao, Z., Eyers, S., Thomas, C., Ellis, N., and Maule, A. 2004. Identification of markers tightly linked to $s b m$ recessive genes for resistance to Pea seed-borne mosaic virus. Theor. Appl. Genet. 109:488-494.

Hughes, S. L., Green, S. K., Lydiate, D. J., and Walsh, J. A. 2002. Resistance to Turnip mosaic virus in Brassica rapa and B. napus and the analysis of genetic inheritance in selected lines. Plant Pathol. 51:567573.

Hwang, J., Li, J., Liu, W. Y., An, S. J., Cho, H., Her, N., Yeam, I., Kim, D., and Kang, B. C. 2009. Double mutations in eIF4E and eIFiso4E confer recessive resistance to Chilli veinal mottle virus in pepper. Mol. Cells 27:329-336.

Jenner, C. E., Keane, G. J., Jones, J. E., and Walsh, J. A. 1999. Serotypic variation in turnip mosaic virus. Plant Pathol. 48:101-108.

Kang, B.-C., Yeam, I., Frantz, J. D., Murphy, J. F., and Jahn, M. M. 2005. The pvrl locus in Capsicum encodes a translation initiation factor eIF4E that interacts with Tobacco etch virus VPg. Plant J. 42:392-405.

Kang, B.-C., Yeam, I., Li, H., Perez, K. W., and Jahn, M. M. 2007. Ectopic expression of a recessive resistance gene generates dominant potyvirus resistance in plants. Plant Biotechnol. J. 5:526-536.

Kanyuka, K., Druka, A., Caldwell, D. G., Tymon, A., Mccallum, N., Waugh, R., and Adams, M. J. 2005. Evidence that the recessive bymovirus resistance locus rym 4 in barley corresponds to the eukaryotic translation initiation factor 4E gene. Mol. Plant Pathol. 6:449-458.

Karimi, M., Inzé, D., and Depicker, A. 2002. GATEWAY vectors for Agrobacterium-mediated plant transformation. Trends Plant Sci. 7:193-195.

Lellis, A. D., Kasschau, K. D., Whitham, S. A., and Carrington, J. C. 2002. Loss-of-susceptibility mutants of Arabidopsis thaliana reveal an essential role for eIF(iso)4E during potyvirus infection. Curr. Biol. 12:1046-1051.

Léonard, S., Plante, D., Wittmann, S., Daigneault, N., Fortin, M. G., and Laliberté, J.-F. 2000. Complex formation between potyvirus VPg and translation eukaryotic initiation factor $4 \mathrm{E}$ correlates with virus infectivity. J. Virol. 74:7730-7737.

Léonard, S., Viel, C., Beauchemin, C., Daigneault, N., Fortin, M. G., and Laliberté, J.-F. 2004. Interaction of VPg-Pro of Turnip mosaic virus with the translation initiation factor $4 \mathrm{E}$ and the poly(A)-binding protein in planta. J. Gen. Virol. 85:1055-1063.

Liu, X. P., Lu, W. C., Liu, Y. K., Wei, S. Q., Xu, J. B., Liu, Z. R., Zhang, H. J., Li, J. L., Ke, G. L., Yao, W. Y., Cai, Y. S., Wu, F. Y., Cao, S. C., Li, Y. H., Xie, S. D., Lin, B. X., and Zhang, C. L. 1996. Occurrence and strain differentiation of turnip mosaic potyvirus and sources of resistance in chinese cabbage in China. Acta Hortic. 407:431-440.

Miyoshi, H., Suehiro, N., Tomoo, K., Muto, S., Takahashi, T., Tsukamoto, T., Ohmori, T., and Natsuaki, T. 2006. Binding analyses for the interaction between plant virus genome-linked protein ( VPg) and plant translational initiation factors. Biochimie 88:329-340.

Monzingo, A. F., Dhaliwal, S., Dutt-Chaudhuri, A., Lyon, A., Sadow, J. H., Hoffman, D. W., Robertus, J. D., and Browning, K. S. 2007. The struc- ture of eukaryotic translation initiation factor-4E from wheat reveals a novel disulfide bond. Plant Physiol. 143:1504-1518.

Naderpour, M., Lund, O. S., Larsen, R., and Johansen, E. 2010. Potyviral resistance derived from cultivars of Phaseolus vulgaris carrying bc-3 is associated with the homozygotic presence of a mutated eIF4E allele. Mol. Plant Pathol. 11: 255-263.

Nicaise, V., Gallois, J.-L., Chafiai, F., Allen, L. M., Schurdi-Levraud, V., Browning, K. S., Candresse, T., Caranta, C., Le Gall, O., and GermanRetana, S. 2007. Coordinated and selective recruitment of eIF4E and eIF4G factors for potyvirus infection in Arabidopsis thaliana. FEBS (Fed. Eur. Biochem. Soc.) Lett. 581:1041-1046.

Plante, D., Viel, C., Léonard, S., Tampo, H., Laliberté, J.-F., and Fortin, M. G. 2004. Turnip mosaic virus VPg does not disrupt the translation initiation complex but interferes with cap binding. Physiol. Mol. Plant Pathol. 64:219-226.

Provvidenti, R., and Hampton, R. O. 1992. Sources of resistance to viruses in the Potyviridae. Arch. Virol. Suppl. 5:189-211.

Rana, D., Van Den Boogaart, T., O'Neill, C. M., Hynes, L., Bent, E., Macpherson, L., Park, J. Y., Lim, Y. P., and Bancroft, I. 2004. Conservation of the microstructure of genome segments in Brassica napus and its diploid relatives. Plant J. 40:725-733.

Rhoads, R. E. 2009. eIF4E: New family members, new binding partners, new roles. J. Biol. Chem. 284:16711-16715.

Robaglia, C., and Caranta, C. 2006. Translation initiation factors: A weak link in plant RNA virus infection. Trends Plant Sci. 11:40-45.

Rodriguez, C. M., Freire, M. A., Camilleri, C., and Robaglia, C. 1998. The Arabidopsis thaliana cDNAs coding for eIF4E and eIF(iso)4E are not functionally equivalent for yeast complementation and are differentially expressed during plant development. Plant J. 13:465-473.

Ruffel, S., Dussault, M.-H., Palloix, A., Moury, B., Bendahmane, A. Robaglia, C., and Caranta, C. 2002. A natural recessive resistance gene against Potato virus $Y$ in pepper corresponds to the eukaryotic initiation factor 4E (eIF4E). Plant J. 32:1067-1075.

Ruffel, S., Gallois, J. L., Lesage, M. L., and Caranta, C. 2005. The recessive potyvirus resistance gene pot-1 is the tomato orthologue of the pepper pvr2-eIF4E gene. Mol. Genet. Genomics 274:346-353.

Ruffel, S., Gallois, J.-L., Moury, B., Robaglia, C., Palloix, A., and Caranta, C. 2006. Simultaneous mutations in translation initiation factors elF4E and $e l F$ (iso) $4 E$ are required to prevent Pepper veinal mottle virus infection of pepper. J. Gen. Virol. 87:2089-2098.

Rusholme, R. L., Higgins, E. E., Walsh, J. A., and Lydiate, D. J. 2007. Genetic control of broad-spectrum resistance to Turnip mosaic virus in Brassica rapa (Chinese cabbage). J. Gen. Virol. 88:3177-3186.

Ruud, K. A., Kuhlow, C., Goss, D. J., and Browning, K. S. 1998. Identification and characterization of a novel cap-binding protein from Arabidopsis thaliana. J. Biol. Chem. 273:10325-10330.

Sato, M., Nakahara, K., Yoshii, M., Ishikawa, M., and Uyeda, I. 2005. Selective involvement of members of the eukaryotic initiation factor $4 \mathrm{E}$ family in the infection of Arabidopsis thaliana by potyviruses. FEBS (Fed. Eur. Biochem. Soc.) Lett. 579:1167-1171.

Schaad, M. C., Anderberg, R. J., and Carrington, J. C. 2000. Strain-specific interaction of the tobacco etch virus Nla protein with the translation initiation factor elF4E in the yeast two-hybrid system. Virology 273:300306.

Shattuck, V. I. 1992. The biology, epidemiology, and control of Turnip mosaic virus. Plant Breed. Rev. 14:199-238.

Walsh, J. A. 1989. Genetic control of immunity to turnip mosaic virus in winter oilseed rape (Brassica napus ssp. oleifera) and the effect of foreign isolates of the virus. Ann. Appl. Biol. 115:89-99.

Walsh, J. A., and Jenner, C. E. 2002. Turnip mosaic virus and the quest for durable resistance. Mol. Plant Pathol. 3:289-300.

Walsh, J. A., Rusholme, R. L., Hughes, S. L., Jenner, C. E., Bambridge, J. M., Lydiate, D. J., and Green, S. K., 2002. Different classes of resistance to turnip mosaic virus in Brassica rapa. Eur. J. Plant Pathol. 108:15-20.

Wittmann, S., Chatel, H., Fortin, M. G., and Laliberté, J.-F. 1997. Interaction of the viral protein genome linked of turnip mosaic potyvirus with the translational eukaryotic initiation factor (iso)4E of Arabidopsis thaliana using the yeast two-hybrid system. Virology 234:84-92.

Zhang, Y.-Y., Li, H.-X., Ouyang, B., and Ye, Z.-B., 2006. Regulation of eukaryotic initiation factor $4 \mathrm{E}$ and its isoform: Implications for antiviral strategy in plants. J. Integr. Plant Biol. 48:1129-1139. 